\title{
SCANNING TUNNELING MICROSCOPY STUDIES OF InGaN GROWTH BY MOLECULAR BEAM EPITAXY
}

\author{
Huajie Chen, ${ }^{*}$ A. R. Smith, ${ }^{*(a)}$ R. M. Feenstra, ${ }^{*}$ D. W. Greve, ${ }^{* *}$ and J. E. Northrup*** \\ *Department of Physics, Carnegie Mellon University, Pittsburgh, PA 15213 \\ **Department of Electrical and Computer Engineering, Carnegie Mellon University \\ ***Xerox Palo Alto Research Center, 3333 Coyote Hill Road, Palo Alto, CA 94304
}

Cite this article as: MRS Internet J. Nitride Semicond. Res. 4S1, G9.5 (1999)

\begin{abstract}
InGaN alloys with indium compositions ranging from $0-40 \%$ have been grown by molecular beam epitaxy. The dependence of the indium incorporation on growth temperature and group III/group V ratio has been studied. Scanning tunneling microscopy images, interpreted using first-principles theoretical computations, show that there is strong indium surface segregation on InGaN. Based on this surface segregation, a qualitative model is proposed to explain the observed indium incorporation dependence on the growth parameters.
\end{abstract}

\section{INTRODUCTION}

Ternary $\operatorname{In}_{\mathrm{x}} \mathrm{Ga}_{1-\mathrm{x}} \mathrm{N}$ alloys are widely used in the development of GaN-based optoelectronic devices. ${ }^{1}$ To achieve high quality layers with desired optical properties, it is important to understand and control the indium incorporation efficiency and uniformity. In conventional III-V semiconductors, surface segregation of metal species ( $\mathrm{Ga}, \mathrm{In}$, or $\mathrm{Al}$ ) is known to be an important process. ${ }^{2}$ In this paper, we present results for the indium incorporation in InGaN during growth by molecular beam epitaxy (MBE), with the characterization performed by x-ray diffraction (XRD), Auger spectroscopy, and scanning tunneling microscopy (STM). The dependence of the indium incorporation on growth parameters is studied. STM images reveal strong surface segregation of the indium, from which a model is proposed to explain the incorporation kinetics.

\section{EXPERIMENT}

The studies described here were performed in a combined MBE/surface analysis system. The growth chamber contains gallium and indium effusion cells, an RF-plasma nitrogen source, and a reflection high energy electron diffraction (RHEED) system. Growth is performed on solvent-cleaned sapphire (0001) substrates, heated first to $700^{\circ} \mathrm{C}$ for $15 \mathrm{~min}$, then exposed at $1050^{\circ} \mathrm{C}$ to the nitrogen plasma with a power level of $550 \mathrm{~W}$ and pressure of $7.0 \times 10^{-5}$ Torr, for $30 \mathrm{~min}$. First a $\mathrm{GaN}$ buffer layer of about $20 \mathrm{~nm}$ thickness is grown at $550^{\circ} \mathrm{C}$ using a nitrogen pressure of $1.5 \times 10^{-5}$ Torr. The growth temperature is then increased to $720^{\circ} \mathrm{C}$ for the remainder of the GaN growth. The total thickness of $\mathrm{GaN}$ was in the range $200-400 \mathrm{~nm}$. Following the $\mathrm{GaN}$ growth, the substrate temperature is lowered to $620-670^{\circ} \mathrm{C}$ for the InGaN deposition. Typical growth rates for the $\mathrm{GaN}$ and $\mathrm{InGaN}$ are $200 \mathrm{~nm} / \mathrm{h}$. Gallium and indium flux rates were calibrated with an in situ crystal thickness monitor. The substrate temperature was measured by a optical pyrometer with emissivity set to be 0.7; care was taken to ensure a clean viewport between the pyrometer and the sample. From our previous work, ${ }^{3}$ it is known that $\mathrm{GaN}$ films prepared in the above manner are nitrogen polar.

\section{RESULTS AND DISCUSSION}




\section{Indium Incorporation Dependence on Growth Parameters}

Several series of samples have been grown with different growth parameters. After the growth, XRD were performed to measure the indium incorporation. For the analysis of the XRD data, we assume that InGaN layer is fully strain relaxed (the thickness of InGaN is typically 200 $\mathrm{nm})$, and Vegard's law applies.

First, we consider variation of the growth temperature, keeping the indium, gallium, and nitrogen flux all constant. Results are shown in Fig. 1. For the set of samples whose XRD results are shown in Fig. 1(a), the indium incident flux is about $22 \%$ of the total metal (that is, In+Ga) flux. It can be seen from the XRD data that indium incorporation decreases when the growth temperature is increased. The XRD study of the sample grown at $660^{\circ} \mathrm{C}$ was performed in triple axis mode to increase the resolution, while the other scans were performed with a $0.8 \mathrm{~mm}$ slit in front of the detector. The fringes seen in the former are the thickness fringes of the GaN layer. A summary of the temperature dependent results is given in Fig. 1(b); an additional set of data is shown there with $\mathrm{In} /(\mathrm{In}+\mathrm{Ga})$ flux ratio of $36 \%$. Both of the data sets show a similar dependence of the indium incorporation on growth temperature. Similar results have been observed in both MOCVD and MBE growth by other groups. ${ }^{4}$
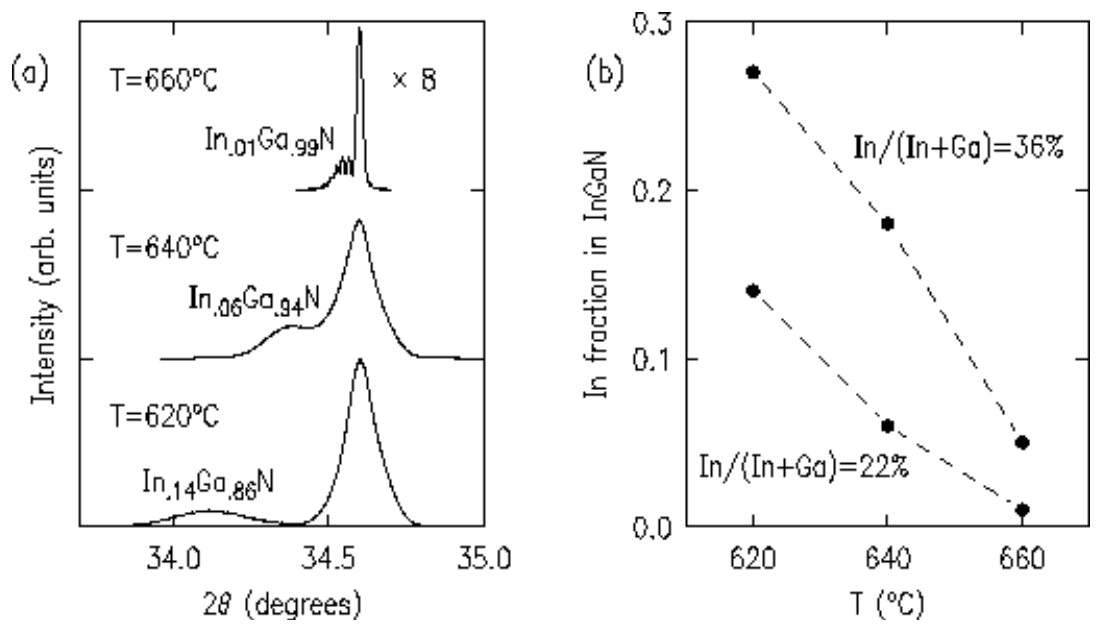

Figure 1. (a) X-ray diffraction $2 \theta-\omega$ scans for a series of $\mathrm{InGaN}$ films grown with a $\mathrm{In} /(\mathrm{In}+\mathrm{Ga})$ flux ratio of $22 \%$. Indium incorporation, deduced from the $\mathrm{x}$-ray results, is indicated for each film. (b) Summary of the indium incorporation dependence on growth temperature.

Let us now consider variation of the group III/group V ratio while keeping the In/Ga ratio constant. The set of samples whose results are shown in Fig. 2(a) were grown at $640^{\circ} \mathrm{C}$, with the $\mathrm{In} /(\mathrm{In}+\mathrm{Ga})$ flux ratio being about $36 \%$. We find that when the metal flux is increased from $3.4 \times$ $10^{14} \mathrm{~cm}^{-2} \mathrm{~s}^{-1}$ to $4.4 \times 10^{14} \mathrm{~cm}^{-2} \mathrm{~s}^{-1}$, indium incorporation decreases from $23 \%$ to $8 \%$. However, comparing the two samples grown with $2.5 \times 10^{14} \mathrm{~cm}^{-2} \mathrm{~s}^{-1}$ and $3.4 \times 10^{14} \mathrm{~cm}^{-2} \mathrm{~s}^{-1}$ metal flux, there is a small increase in indium incorporation with increasing metal flux. For the sample grown with $4.4 \times 10^{14} \mathrm{~cm}^{-2} \mathrm{~s}^{-1}$ metal flux, there is an XRD peak at $32.9^{\circ}$, arising from indium droplet (101) planes which are parallel to the $\mathrm{GaN}(000 \overline{1})$ planes. The results are summarized in Fig. 2(b); two additional sets of data are shown there with different growth temperatures.

Also indicated in Fig. 2(b) is a dashed line indicating the transition point between $\mathrm{N}$ rich and group III rich growth regimes. This transition point is obtained from studies of GaN growth in the absence of indium, ${ }^{5}$ where a transition from rough to smooth morphology (i.e. spotty to streaky RHEED pattern) is found as a function of gallium flux. In the present work, using the same nitrogen pressure as for the InGaN growth and a temperature of $720^{\circ} \mathrm{C}$, we find this transition gallium flux to be about $2.9 \times 10^{14} \mathrm{~cm}^{-2} \mathrm{~s}^{-1}$. This value varies slightly between the data sets in Fig. 2(b), because the nitrogen flux varies slightly from set to set. For the case of InGaN growth, we find that there is no such clear transition from rough to smooth growth, so we use the 
transition flux determined from the GaN growth as an approximate reference. From Fig. 2(b), we see that in the nitrogen rich regime the indium incorporation increases when the metal flux is increased. In contrast, we find in the group III rich region that the indium incorporation decreases when the metal flux is increased. We actually have much more data in this region, and they all show the same trend. This behavior is different from what have been reported for MOCVD growth, ${ }^{6}$ where indium incorporation consistently increases when metal flux is increased.
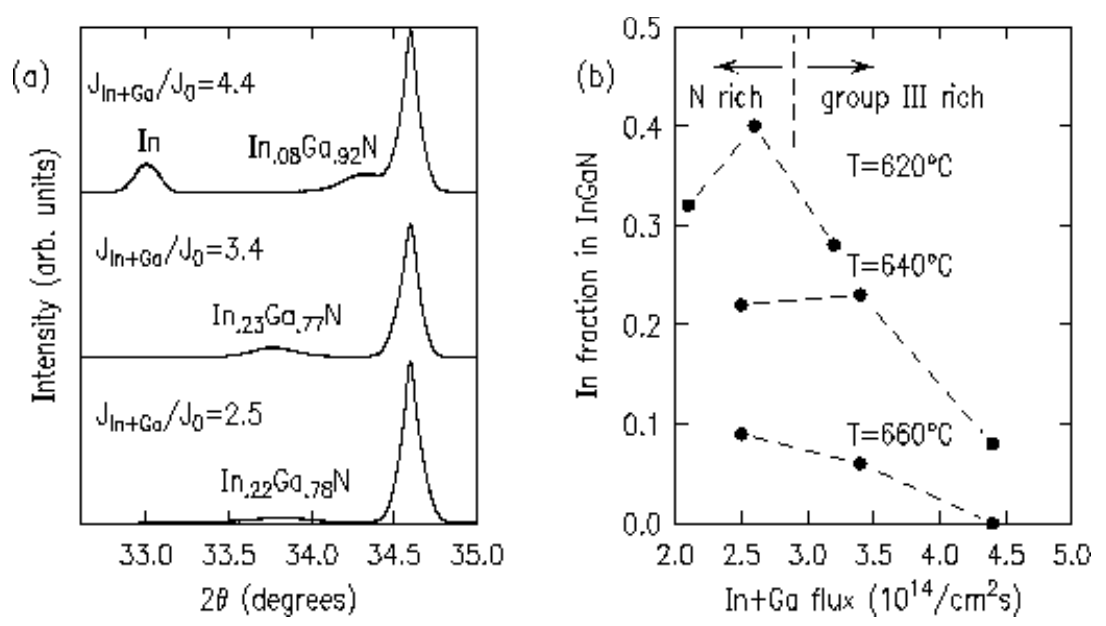

Figure 2. (a) X-ray diffraction $2 \theta-\omega$ scans for a series of InGaN films grown with varying In+Ga flux, $\mathrm{J}_{\mathrm{In}+\mathrm{Ga}}$, with $\mathrm{J}_{0}=10^{14}$ $\mathrm{cm}^{-2} \mathrm{~s}^{-1}$, at $640^{\circ} \mathrm{C}$. The $\mathrm{In} /(\mathrm{In}+\mathrm{Ga})$ flux ratio was kept constant at $36 \%$. (b) Summary of the indium incorporation dependence on $(\mathrm{In}+\mathrm{Ga})$ flux.

\section{$\underline{\text { Indium Surface Segregation }}$}

Figure 3 shows an STM image of an InGaN surface, prepared by growth at $670^{\circ} \mathrm{C}$ with $\mathrm{In} /(\mathrm{In}+\mathrm{Ga})$ flux ratio of $20 \%$. As mentioned earlier, the samples we studied here are nitrogen polar, in which case we know that the surface layer consists of an adlayer of metal atoms (gallium and/ or indium). ${ }^{7}$ An XRD scan of this sample showed no InGaN peak, meaning that there is negligible indium incorporation in the bulk. In the middle of the STM image, there is a step, and the image shows two separate terraces. Each terrace displays two types of regions, one bright and the other dark. In this type of STM constant-current image, roughly speaking, the bright areas are higher in morphology and dark areas are lower. As can be seen from the line cut, the height difference between bright and dark area is $0.30 \pm 0.05 \AA$. As described below, we identify the bright areas to be indium covered regions and dark areas to be gallium covered regions.

For the sample imaged in Fig. 3, Auger spectroscopy showed that there is a significant indium signal, with a In/Ga signal ratio of about 0.5. Although our Auger system is not well calibrated, so quantitative determination of surface concentration is difficult, the result certainly shows that there is a significant amount of indium on the surface. Furthermore, theoretical computations have been performed which permit the identification of the bright and dark areas in the STM image. Those results are pictured in Fig. 4. The total energy calculation is performed within the local density functional theory using first-principles pseudopotential methods for various $\mathrm{GaN}(000 \overline{1}) 1 \times 1: \mathrm{Ga}_{\mathrm{y}} \mathrm{In}_{1-\mathrm{y}}$ adlayer structures. The calculations are performed in a $2 \times 2$ unit cell with various numbers of In and $\mathrm{Ga}$ atoms in the top site. The equilibrium adlayer In-N bond length is found to be about $2.23 \AA$ while the equilibrium Ga-N bond in the adlayer is $1.99 \AA$. These values are essentially independent of $y$. From explicit examination of charge density contours for states located near the Fermi-level, we find that the different height of the adatoms above the surface $(\sim 0.2 \AA)$ is manifested in a corrugation in the charge density of a similar magnitude at a height of several $\AA$ above the adatoms. Based on these two reasons, and on our previous experience on the study of GaN surface, we identify the bright regions as arising from indium atoms in the surface adlayer, and the dark regions as arising from gallium atoms in the 
adlayer.
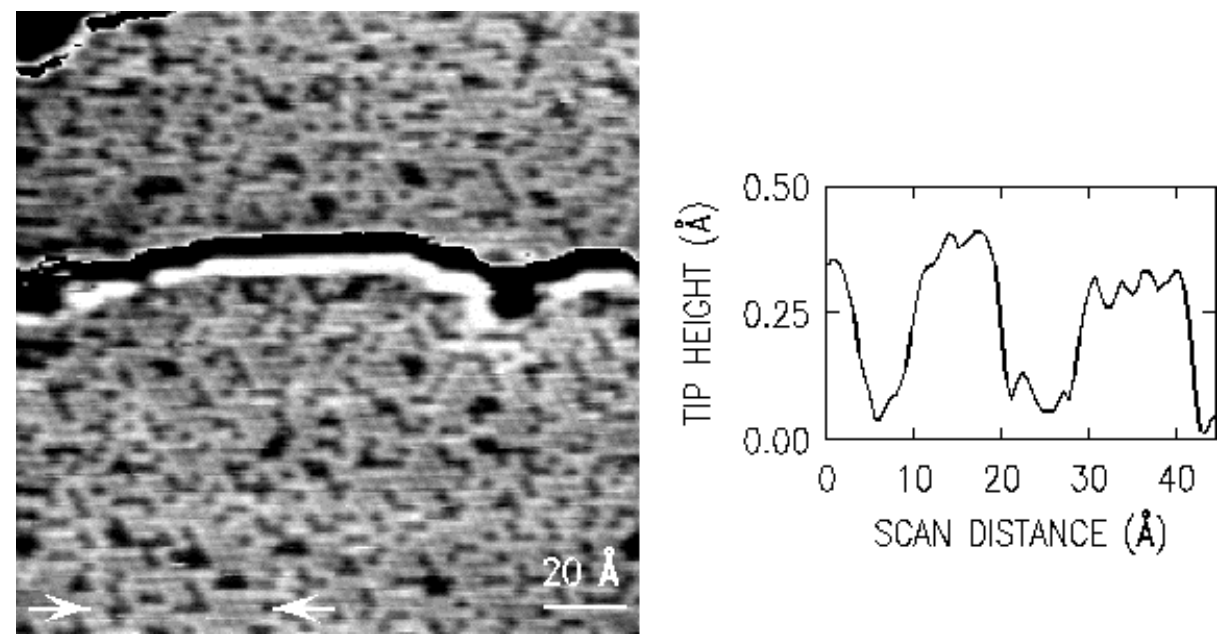

Figure 3. STM image of the $\operatorname{InGaN}(000 \overline{1})$ surface, obtained from a sample with negligible indium incorporation in the bulk. The image was acquired with sample bias voltage of $-0.5 \mathrm{~V}$ and tunnel current of $0.075 \mathrm{nA}$. The grey scale range is $0.6 \AA$ for each terrace. A line cut, taken at the position of the arrows in the figure, is shown on the right side of the figure.
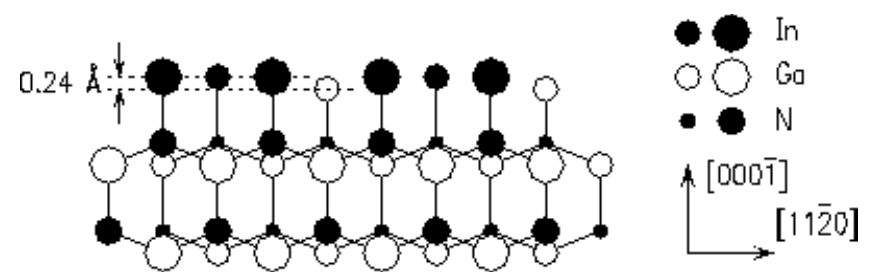

Figure 4. Schematic view of the InGaN surface in an $(1 \overline{1} 00)$ projection, showing theoretical results for atomic positions. The surface adlayer consists of $75 \%$ In plus $25 \% \mathrm{Ga}$, in a $2 \times 2$ arrangement..

From the STM image of Fig. 3 it is clear that the InGaN surface is mostly covered with indium atoms, whereas for the same sample there is negligible indium incorporation in the bulk. Thus there is strong surface segregation of indium on the InGaN. We have observed similar surface segregation on other samples, including those containing significant amounts of indium in the bulk. Indium surface segregation has been attributed in large part ${ }^{8}$ to the fact that the InN bond is weaker than the GaN bond (1.93 eV for InN and $2.24 \mathrm{eV}$ for $\mathrm{GaN}){ }^{9}$ In the bulk, a metal atom forms four bonds with nitrogen atoms, whereas on the surface, for N-polar InGaN, a metal atom forms only one bond with a nitrogen atom. Therefore it is energetically favorable for a gallium atom on the surface to switch sites with an indium atom in the bulk.

\section{$\underline{\text { Model }}$}

Figure 5 illustrates the near surface region of a N-polar InGaN film, showing a typical distribution of metal atoms (indium and gallium) on the surface and in the bulk. From prior studies in the absence of indium, it is known that the surface is terminated with a monolayer of metal atoms (layer 2 in Fig. 5), and a few additional metal atoms (layer 1) may reside on top of this adlayer. ${ }^{7}$ The population of metal atoms in layer 1 depends on how metal rich the growth is. For GaN growth, it is known that the gallium atoms in layer 1 diffuse quickly, ${ }^{10,11}$ and the observation of step flow growth ${ }^{12,13}$ implies that the gallium atoms (and presumably also the nitrogen atoms) diffuse to surface steps where they incorporate. For the present case of InGaN growth, the observed surface segregation reveals that layer 2 contains mainly indium atoms. This 
surface composition will affect the growth in several ways. First, the atomic diffusivity will increase on an indium covered (000) $)$ surface compared with a gallium covered surface. ${ }^{14}$ Second, a gallium atom in layer 1 may simply switch sites with an indium atom in layer 2 without diffusing to a step edge, and that particular gallium atom could possibly further switch positions with an indium atom in the bulk. Therefore the growth kinetics for Ga incorporation in InGaN growth may be different from that in $\mathrm{GaN}$ growth. Third, if the nitrogen source is $\mathrm{NH}_{3}$, rather than an RF-plasma source, the decomposition rate of $\mathrm{NH}_{3}$ may be affected by the surface metal species.

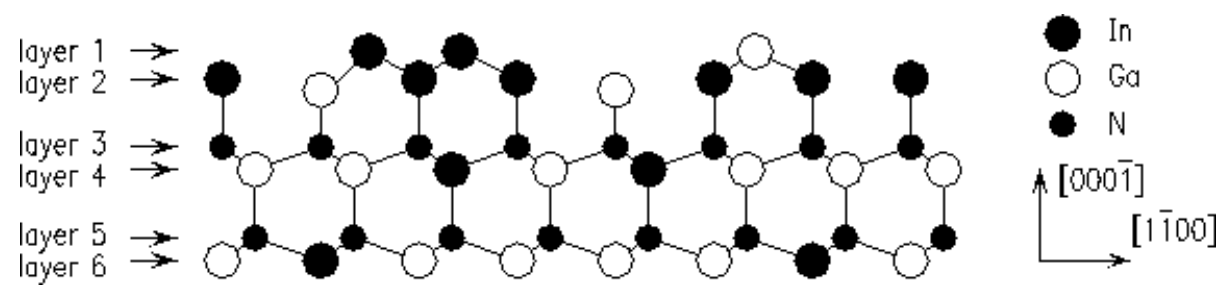

Figure 5. Schematic view of the $\operatorname{InGaN}(000 \overline{1})$ surface layers, in a $(11 \overline{2} 0)$ projection: layer 1 , the metal adatoms residing on top of the metal adlayer; layer 2, metal adlayer on nitrogen; layers 3 and 5, nitrogen atoms; layers 4 and 6, metal atoms.

Since there is a strong surface segregation of indium atoms, the gallium atom population on the surface is small, under the condition that the growth is not overly metal rich. Then, the gallium evaporation will also be small, and most of the incident gallium flux will incorporate into the bulk. For indium atoms, the incident flux can proceed in one of several kinetic pathways. One is to be incorporated into the bulk. The second possibility is to evaporate, including evaporation from layer 2 (which is actually decomposition of $\mathrm{InN}$ ) or evaporation from layer 1 (the rate of which depends on the numbers of atoms in that layer). We denote the concentration of indium atoms in the $\mathrm{i}$ th layer as $\left[\mathrm{In}_{i}\right]$, and the rate of indium evaporation from the $\mathrm{i}$ th layer as $\mathrm{R}_{\mathrm{In} i}$. When the metal flux is increased while keeping the nitrogen flux constant, the metal atom population in layer 1 will increase and so $R_{\mathrm{In} 1}$ will increase to keep the metal flux in balance. When the metal flux is further increased, the layer 1 metal population is so high that metal droplets will form, which is the third pathway for the incident indium.

Based on this model, the dependence of indium incorporation on group III/group V ratio which was described in the earlier section can be explained. In the metal rich region, the maximum amount of In+Ga which can be incorporated into the bulk is limited by the active nitrogen flux. When the metal flux is increased, those additional gallium atoms will compete to go into bulk. Since there is a strong indium surface segregation, those additional gallium atoms will mostly go into bulk and kick out indium atoms, so that the indium incorporation will decrease. Excess indium atoms, formed from the increased metal flux as well as the decreased bulk incorporation, will tend to increase $\left[\mathrm{In}_{1}\right]$, and so $\mathrm{R}_{\mathrm{In} 1}$ will increase. Thus, the excess indium will evaporate, or alternatively, it will form droplets if there is too much indium. In the nitrogen rich region, however, the number of metal atoms in layer 1 is minimal. Thus, metal evaporation proceeds mainly via $\mathrm{R}_{\operatorname{In} 2}$, which is almost constant for a given temperature. Therefore with the loss of indium being constant, an increase of both indium and gallium flux while keeping their ratio constant will lead to an increase in the indium incorporation.

\section{CONCLUSION}

The dependence of indium incorporation on temperature and metal flux has been studied during MBE growth of $\mathrm{InGaN}$. It is found that the incorporation decreases when the growth temperature is increased, it decreases when the group III/group V flux ratio is increased under 
metal rich conditions, and it increases as a function of this flux ratio under nitrogen rich conditions. STM images show that InGaN surface is mostly covered with indium atoms, revealing a strong surface segregation of the indium. Based on this surface segregation, a model is proposed to explain the indium incorporation dependence on the growth parameters.

\section{ACKNOWLEDGEMENTS}

This work was supported by grants from the National Science Foundation, DMR-9615647, and from the Office of Naval Research, N00014-96-1-0214.

(a) Present address: Dept. of Physics and Astronomy, Ohio University, Athens, Ohio 45701.

\section{REFERENCES}

[1] S. Nakamura, M. Senoh, S.-I. Nagahama, N. Iwasa, T. Yamada, T. Matsushita, H. Kiyoku, Y. Sugimoto, T. Kozaki, H. Umemoto, M. Sano, and K. Chocho, Jpn. J. Appl. Phys., Part 2 36, L1568 (1997); S. Nakamura and G. Fasol, The Blue Laser Diode (Springer, Berlin, 1997).

[2] J.M. Moison, C. Guille, F. Houzay, F. Barthe, and M. Van Rompay, Phys. Rev. B 40, 6149 (1989).

[3] A.R. Smith, R.M. Feenstra, D.W. Greve, M.-S. Shin, M. Skowronski, J. Neugebauer, J.E. Northrup, Appl. Phys. Lett. 72, 2114 (1998).

[4] See for example, S. Nakamura, Microelectron. J. 25, 651 (1994); J.M. Van Hove, P.P. Chow, A.M. Wowchak, J.J. Klaassen, R. Hickman II, and C. Polley, J. Vac. Sci. Technol. B 16, 1286 (1998); N. Grandjean, and J. Massies, Appl. Phys. Lett. 72, 1078 (1998).

[5] A.R. Smith, V. Ramachandran, R.M. Feenstra, D.W. Greve, A. Ptak, T. Myers, W. Sarney, L. Salamanca-Riba, M. Shin, and M. Skowronski, MRS Internet J. Nitride Semicond. Res. 3,12 (1998).

[6] E.L. Piner, F.G. Mcintosh, J.C. Roberts, K.S. Boutros, M.E. Aumer, V.A. Joshkin, N.A. ElMasry, S.M. Bedair, and S.X. Liu, Mat. Res. Soc. Symp. Proc. 449, 85 (1997).

[7] A.R. Smith, R.M. Feenstra, D.W. Greve, J. Neugebauer, and J.E. Northrup, Phys. Rev. Lett. 79, 3934 (1997).

[8] J.E. Northrup, J. Neugebauer, and L.T. Romano, submitted to Appl. Phys. Lett.

[9] W.A. Harrison, Electronic structure and the properties of solids (Freeman, San Francisco, 1980) p 176.

[10] A.R. Smith, R.M. Feenstra, D.W. Greve, J. Neugebauer, and J.E. Northrup, Appl. Phys. A 66, S947 (1998).

[11] T. Zywietz, J. Neugebauer, and M. Scheffler, Appl. Phys. Lett. 73, 487 (1998).

[12] E.J. Tarsa, B. Heying, X.H. Wu, P. Fini, S.P. DenBaars, and J.S. Speck, J. Appl. Phys. 82, 5472 (1997).

[13] A.R. Smith, V. Ramachandran, R.M. Feenstra, D.W. Greve, M.-S. Shin, M. Skowronski, J. Neugebauer, J.E. Northrup, J. Vac. Sci. Technol. A 16, 1641 (1998).

[14] T.K. Zywietz, J. Neugebauer, M. Scheffler, and J.E. Northrup (unpublished). 